\title{
Control of glyphosate resistant giant ragweed in soybean with preplant herbicides
}

\author{
Joanna Follings ${ }^{1}$, Nader Soltani ${ }^{1^{*}}$, Darren E. Robinson ${ }^{1}$, François J. Tardif ${ }^{2}$, Mark B. Lawton ${ }^{3}$, \\ Peter H. Sikkema ${ }^{1}$ \\ ${ }^{1}$ University of Guelph Ridgetown Campus, Ridgetown, Canada; ${ }^{*}$ Corresponding Author: soltanin@uoguelph.ca \\ ${ }^{2}$ University of Guelph, Guelph, Canada \\ ${ }^{3}$ Monsanto Canada Inc., Guelph, Canada \\ Received 1 February 2013; revised 15 March 2013; accepted 20 April 2013
}

Copyright (C) 2013 Joanna Follings et al. This is an open access article distributed under the Creative Commons Attribution License, which permits unrestricted use, distribution, and reproduction in any medium, provided the original work is properly cited.

\begin{abstract}
Giant ragweed was the first glyphosate resistant weed identified in Canada. It is a very competetive weed in row crop production and has been found to drastically reduce yields of soybean; therefore, control of this competitive weed is essential. The objective of this study was to determine effective control options for glyphosate resistant giant ragweed in soybean with herbicides applied preplant. Eighteen herbicide combinations were evaluated in field studies conducted in 2011 and 2012 at five locations with confirmed glyphosate resistant giant ragweed. Glyphosate plus 2,4-D ester or amitrole provided the best control of glyphosate resistant giant ragweed 4 WAA. Glyphosate plus 2,4-D ester provided 98 to $99 \%$ control and was equivalent to the weed free check at all locations. Glyphosate plus amitrole provided $90 \%$ to $93 \%$ control and was equivalent to the weed free check at 4 of 5 locations. Herbicides providing residual activity provided variable control across all locations. Of the herbicides with residual activity evaluated, glyphosate plus linuron provided the best control of glyphosate resistant giant ragweed; however, control was inconsistent across locations and years. Glyphosate plus linuron provided $23 \%$ to $99 \%$ control and was equal to the weed free check at one location 8 WAA.
\end{abstract}

Keywords: Chlorimuron-Ethyl; Cloransulam-Methyl; Flumioxazin; Herbicide-Resistant Weeds; Imazethapyr; Linuron; Metribuzin; Saflufenacil/Dimethenamid-P; Soybean

\section{INTRODUCTION}

Glyphosate is a nonselective herbicide that inhibits the enzyme 5-enolpyruvylshikimate-3-phosphate synthase (EPSPS) [1,2]. The inhibition of EPSPS stops plants from synthesizing certain aromatic acids that are essential for plant growth [2]. Glyphosate has low environmental and mammalian impact. There are various properties of glyphosate that make it a safe herbicide. The EPSPS enzyme can only be found in plants, bacteria and fungi [2]. Therefore, glyphosate has a low toxicity for non-target organisms such as mammals, birds and fish.

Glyphosate has limited mobility and is rapidly degraded in the soil. Glyphosate is rapidly inactivated when applied to the soil due to adsorption to clay and organic matter through the phosphonic acid moiety [3]. In addition to having minimal activity in the soil glyphosate is also unlikely to evaporate from the soil surface due to its low volatility [4]. It is because of these properties that glyphosate does not provide residual control of weeds.

There has been rapid adoption of glyphosate resistant crops since the introduction of glyphosate resistant soybean in 1996 [5]. The use of glyphosate resistant crops has resulted in changes in crop production systems. Since the introduction of glyphosate resistant crops there has been an increase in conservation tillage and a concomitant decrease in the use of herbicides with a different mode of action [6]. These practices impact selection pressure and have facilitated weed population shifts and the selection of glyphosate resistant weeds [6].

It was previously thought that glyphosate resistance was very unlikely to occur in weeds. Some of the reasons for the remote possibility of glyphosate resistant weeds included glyphosate's unique mode of action, limited uptake from the soil and rapid degradation in the soil [7]. However, there is wide spread glyphosate resistant monocot and dicot weeds in many agricultural producing areas in the world. In 1996 the first glyphosate resistant weed was reported [8]. A population of rigid ryegrass (Loliumrigidum) was found to have a 7- to 11-fold resis- 
tance level to glyphosate after application of glyphosate two to three times annually in an Australian orchard for 15 years [8]. Subsequently, additional reports of glyphosate resistant weeds were reported. Currently there are 24 weed species resistant to glyphosate [9].

Ambrosia trifida L., commonly known as giant ragweed, is found in cultivated fields and orchards as well as non-cropped environments such as roadside ditches and river banks [10]. Giant ragweed is a member of the composite family and is an erect annual broadleaf weed [10]. Giant ragweed has a long emergence period. Seedlings begin to emerge in early March [11] and continue to emerge until late July [12]. Giant ragweed's long emergence period and its ability to grow rapidly make it a very competitive weed in row crop production. A mature giant ragweed plant can grow up to $6 \mathrm{~m}$ in height depending on competition for sunlight $[10,13]$. Giant ragweed has been found to drastically reduce yields of soybean. Baysinger and Sims [14] reported a 92\% yield loss in soybean with a giant ragweed density of 16 plants per $9 \mathrm{~m}$ of row. Soybean growers historically controlled this competitive weed with glyphosate; however, in 2008 a giant ragweed accession from Essex County in Ontario was confirmed to be resistant to glyphosate making it the first glyphosate resistant weed in Canada [9].

Glyphosate resistant giant ragweed is an increasing problem in glyphosate resistant cropping systems in Ontario. As of 2010 there were 48 locations confirmed with glyphosate-resistant giant ragweed in Ontario [15]. There are few herbicide options for glyphosate-resistant giant ragweed control in soybean. Vink et al. [15] reported that glyphosate plus 2,4-D provided $97 \%$ to $99 \%$ control of glyphosate-resistant giant ragweed. Control with glyphosate plus cloransulam-methyl or saflufenacil provided variable control at $68 \%$ to $100 \%$ and $71 \%$ to $94 \%$, respectively. Research is required to identify additional weed management options for this competitive weed in soybean. Therefore, the objective of this study was to determine new herbicide options for the control of glyphosate resistant giant ragweed in soybean with herbicides applied preplant.

\section{MATERIALS AND METHOD}

\subsection{Study Establishment}

Field studies were conducted in 2011and 2012 at five locations with confirmed glyphosate resistant giant ragweed. The field sites were located near Windsor (L2 and L5), La Salle (L1 and L4) and Amherstburg (L3), Ontario. Two sets of experiments evaluating the effectiveness of glyphosate tankmixes with herbicides applied preplant were conducted which are referred to as "enhanced burndown" and "burndown plus residual". Soil texture, soil organic matter content, soil $\mathrm{pH}$, soybean cultivar, seeding date, seeding rate, row spacing, herbicide application date and giant ragweed height are presented in Table 1.

Experiments were set up in a randomized complete block design with four replications. Each plot was $8 \mathrm{~m}$ long and $2.5 \mathrm{~m}$ wide. The first experiment (enhanced burndown) evaluated herbicides applied preplant (PP) that provided limited or no residual control. Herbicides evaluated in this study are listed in Tables 2-5. The second experiment (burndown plus residual) evaluated herbicides applied PP that provided burndown plus residual control. Herbicides evaluated in this study are listed in Tables 6-10. The herbicide rates used were the maximum labeled rate registered for use in Ontario. A weedy and weed-free check was included in each experiment. All weed-free check plots were maintained with 2,4-D ester $\left(500 \mathrm{~g}\right.$ a.e. $\left.\mathrm{ha}^{-1}\right)$ plus glyphosate $\left(900 \mathrm{~g}\right.$ a.e. $\left.\mathrm{ha}^{-1}\right)$ applied $\mathrm{PP}$ and subsequent hand hoeing as required.

Herbicide treatments were applied with a $\mathrm{CO}_{2}$-pressurized backpack sprayer equipped with ULD 120-02 flat fan nozzles (Hypro, New Brighton, MN) calibrated to deliver $200 \mathrm{~L} \cdot \mathrm{ha}^{-1}$ of water at $210 \mathrm{kPa}$. Herbicide treatments were applied with a 1.5 meter boom with four nozzles spaced $50 \mathrm{~cm}$ apart over the centre of the plot. Herbicide treatments were applied when giant ragweed reached 15 $\mathrm{cm}$ in height (Table 1).

\subsection{Data Collection}

Percent weed control was rated 1, 2 and 4 WAA and 1,

Table 1. Location and soil characteristics, soybean variety, planting date, soybean population, herbicide application date, and giant ragweed height at time of application for experiments conducted in 2011 and 2012 in Ontario, Canada.

\begin{tabular}{|c|c|c|c|c|c|c|c|c|c|}
\hline Location & Year & Soil Texture & Soil OM \% ${ }^{a}$ & Soil pH & Soybean Variety & Planting Date & $\begin{array}{l}\text { Soybean } \\
\text { Population }\left(\text { seeds } \cdot \mathrm{ha}^{-1}\right)\end{array}$ & $\begin{array}{c}\text { Herbicide } \\
\text { ) Application Date }\end{array}$ & $\begin{array}{c}\text { Giant Ragweed } \\
\text { Height }(\mathrm{cm})\end{array}$ \\
\hline 1-LaSalle & 2011 & Loam & 2.6 & 7.5 & Dekalb 31-10 & June 13 & 467,029 & May 21 & $1-9$ \\
\hline 2-Windsor & 2011 & Loam & 2.8 & 6.9 & Pioneer 92Y80 & June 15 & 420,079 & June 2 & $4-12$ \\
\hline 3-Amherstburg & 2012 & Clay Loam & 3.7 & 7.9 & Pioneer 92Y53 & May 22 & 568,100 & May 1 & $2-8$ \\
\hline 4-LaSalle & 2012 & Loam & 3.1 & 7.3 & Dekalb 21-11 & May 16 & 444780 & May 8 & $4-13$ \\
\hline 5-Windsor & 2012 & Clay Loam & 4.6 & 6.6 & Pioneer 93 Y05 & June 8 & 432,250 & May 8 & $2-10$ \\
\hline
\end{tabular}


2, 4 and 8 WAA in the enhanced burndown and burndown plus residual experiments, respectively. Weed control was rated visually on a scale of $0 \%$ to $100 \%$, where $0 \%$ was no control of giant ragweed compared to the weedy check and $100 \%$ was complete control of giant ragweed. At each control rating giant ragweed height and density (plants per two $0.25 \mathrm{~m}^{2}$ quadrats) were recorded. At 4 WAA, giant ragweed density and biomass was determined in each plot by counting giant ragweed plants in two $0.25 \mathrm{~m}^{2}$ quadrats. Giant ragweed plants were cut off at the soil surface from the two quadrats, placed in bags, dried at $60^{\circ} \mathrm{C}$ to a constant moisture content and the dry weights were recorded. Soybean injury was rated 1, 2, 4 and 8 WAA. Soybean injury was rated visually on a scale of $0 \%$ to $100 \%$, where $0 \%$ was no soybean injury and $100 \%$ was soybean death. At crop maturity, soybeans were hand harvested from $2 \mathrm{~m}$ of row from each plot at all locations. Soybeans were threshed in a stationary thresher and the weight and moisture were recorded. Yields were adjusted to $13.5 \%$ moisture.

\subsection{Statistical Analysis}

An analysis of variance was conducted on all data using the PROC MIXED procedure in SAS (Ver. 9.2, SAS Institute Inc., Cary, NC). Variances were separated into the random effects of location (year and location), replication (at each location) and location by treatment. Herbicide treatment was considered the fixed effect. The significance of the random effects (location, replication and location by treatment) and their interaction with fixed effects was tested using the Z-test of the variance estimate. The significance of the fixed effects was tested using the F-test. Significant location by treatment interactions were found for all variables; therefore, locations were analyzed according to their interaction and presented accordingly. To ensure the assumptions (errors are independent, homogenous and normally distributed) of the variance analysis were met; residual plots were examined. Data were tested for normality using the Shapiro-Wilk statistic as generated by the UNIVARIATE procedure in SAS. If necessary, a transformation of the data (natural log, square root or arcsine square root) was applied and chosen based on the highest Shapiro-Wilk statistic generated. The means between treatments were produced and separated using Fisher's protected LSD at $\mathrm{P}<0.05$.

\section{RESULTS AND DISCUSSION}

\subsection{Enhanced Burndown}

The herbicides evaluated in the enhanced burndown experiment did not cause any injury in soybean (data not shown).

For control 1 WAA, data were analyzoped separately in groups L1, L2, L3, L4, and L5. At 1 WAA, all the herbicides evaluated provided better control of glyphosate resistant giant ragweed compared to glyphosate alone. Control with glyphosate ranged from $31 \%$ to $43 \%$ at 1 WAA (Table 2). Glyphosate plus paraquat, saflufenacil, or saflufenacil/dimethenamid-p were the most effective treatments 1 WAA providing $85 \%$ to $98 \%, 87 \%$ to $96 \%$ and $89 \%$ to $96 \%$ control, respectively (Table 2). Glyphosate plus 2,4-D ester or glufosinate provided up to 91\% and 89\% control, respectively (Table 2). Glyphosate plus amitrole, carfentrazone, cloransulam-methyl, chlorimuron-ethyl, flumioxazin, or chlorimuron-ethyl plus flumioxazin provided less than $80 \%$ control across all locations (Table 2).

For control 2 WAA, data was analyzed separately in groups L1, L2, L3, L4, and L5. Glyphosate provided only $29 \%$ to $35 \%$ control (Table 3 ). The most effective treatment was glyphosate plus 2,4-D ester which provided $83 \%$ to $94 \%$ control (Table 3). This is similar to findings of Vink et al. [15] who reported $80 \%$ to $95 \%$ control with glyphosate plus 2,4-D ester applied at $900 \mathrm{~g}$ a.e. $\mathrm{ha}^{-1}+500 \mathrm{~g}$ a.e. ha ${ }^{-1} 2$ WAA. Glyphosate plus amitrole, glufosinate, paraquat, saflufenacil, saflufenacil/ dimethenamid-p, or cloransulam-methyl provided up to $86 \%, 93 \%, 98 \%, 91 \%, 92 \%$ and $85 \%$ control, respectively (Table 3). Glyphosate plus carfentrazone, chlorimuron-ethyl, flumioxazin or chrlorimuron-ethyl plus flumioxazin provided less than $80 \%$ control across all locations (Table 3).

At 4 WAA L2 and L5, L3 and L4 could be combined while L1, was analyzed separately. Glyphosate provided $26 \%$ to $39 \%$ control 4 WAA (Table 4). Glyphosate plus 2,4-D was the most effective treatment 4 WAA providing $98 \%$ to $99 \%$ control and was equivalent to the weed free control across all locations (Table 4). This is similar to the findings of Vink et al. [15] who reported $97 \%$ to $98 \%$ control with glyphosate plus 2,4-D ester applied at $900 \mathrm{~g}$ a.e. $\mathrm{ha}^{-1}+500 \mathrm{~g}$ a.e. ha ${ }^{-1} 28$ DAA. Glyphosate plus amitrole provided 90 to $93 \%$ control (Table 4). At L2 and L5 and L3 and L4, glyphosate plus amitrole was equivalent to the weed free control (Tables 3 and 4). At 4 WAA glyphosate plus paraquat provided $57 \%$ to $84 \%$ control (Table 4). At L2 and L5, glyphosate plus paraquat was equivalent to the weed free control and provided less than $80 \%$ control at L1 and L3 and L4 (Table 4). Glyphosate plus cloransulam-methyl provided 62 to $94 \%$ control (Table 4). This is consistent with the findings of Vink et al. [15] who reported $68 \%$ to $97 \%$ control with glyphosate plus cloransulam-methyl applied at $900 \mathrm{~g}$ a.e. $\mathrm{ha}^{-1}+17.5 \mathrm{~g}$ a.i. $\mathrm{h}^{-1}$. Glyphosate plus saflufenacil or saflufenacil/dimethenamid-p provided $46 \%$ to $91 \%$ and $47 \%$ to $93 \%$ control, respectively (Table 4). Soltani et al. [16] reported $45 \%$ to $80 \%$ and $53 \%$ to $63 \%$ control with saflufenacil at $75 \mathrm{~g}$ a.i. $\mathrm{ha}^{-1}$ and saflufenacil/dimethena- 
Table 2. Percent control of glyphosate resistant giant ragweed 1 WAA for the "enhanced burndown" experiments conducted in 2011 and 2012 in Ontario, Canada.

\begin{tabular}{|c|c|c|c|c|c|c|}
\hline \multirow[b]{2}{*}{ Treatment } & \multirow[b]{2}{*}{ Rate (g a.i. ha $\left.{ }^{-1}\right)$} & \multicolumn{5}{|c|}{ Percent Control 1 WAA $^{\mathrm{z}}$} \\
\hline & & $\mathrm{L}^{\mathrm{z}}$ & $\mathrm{L} 2$ & L3 & L4 & L5 \\
\hline Weedy Check & & $0^{\mathrm{h}}$ & $0^{\mathrm{k}}$ & $0^{\mathrm{h}}$ & $0^{\mathrm{h}}$ & $0^{\mathrm{g}}$ \\
\hline Weed Free Check & & $100^{\mathrm{a}}$ & $100^{\mathrm{a}}$ & $100^{\mathrm{a}}$ & $100^{\mathrm{a}}$ & $100^{\mathrm{a}}$ \\
\hline Glyphosate & 900 & $43^{\mathrm{g}}$ & $39^{\mathrm{j}}$ & $32^{\mathrm{g}}$ & $31^{\mathrm{g}}$ & $38^{\mathrm{f}}$ \\
\hline Glyphosate + Amitrole & $900+2000$ & $64^{\mathrm{e}}$ & $74^{\mathrm{g}}$ & $51^{\mathrm{f}}$ & $47^{\mathrm{f}}$ & $40^{\mathrm{f}}$ \\
\hline Glyphosate $+2,4$-D Ester & $900+500$ & $91^{\mathrm{bc}}$ & $84^{\mathrm{e}}$ & $77^{\mathrm{d}}$ & $81^{\mathrm{c}}$ & $82^{c}$ \\
\hline Glyphosate + Carfentrazone & $900+17.5$ & $75^{\mathrm{d}}$ & $65^{\mathrm{hi}}$ & $74^{d}$ & $74^{\mathrm{d}}$ & $71^{\mathrm{d}}$ \\
\hline Glyphosate + Glufosinate & $900+500$ & $86^{\mathrm{c}}$ & $89^{\mathrm{d}}$ & $87^{\mathrm{c}}$ & $82^{\mathrm{c}}$ & $81^{\mathrm{c}}$ \\
\hline Glyphosate + Paraquat & $900+1100$ & $85^{\mathrm{c}}$ & $98^{\mathrm{b}}$ & $93^{\mathrm{b}}$ & $88^{\mathrm{b}}$ & $95^{\mathrm{b}}$ \\
\hline Glyphosate + Saflufenacil ${ }^{\mathrm{y}}$ & $900+25$ & $94^{\mathrm{b}}$ & $96^{c}$ & $93^{\mathrm{b}}$ & $87^{\mathrm{b}}$ & $94^{\mathrm{b}}$ \\
\hline Glyphosate + Saflufenacil/Dimethenamid- $\mathrm{p}^{\mathrm{x}}$ & $900+245$ & $95^{\mathrm{b}}$ & $96^{c}$ & $94^{\mathrm{b}}$ & $89^{\mathrm{b}}$ & $91^{\mathrm{d}}$ \\
\hline Glyphosate + Cloransulam-methyl ${ }^{\mathrm{w}}$ & $900+17.5$ & $77^{\mathrm{d}}$ & $79^{\mathrm{f}}$ & $34^{\mathrm{g}}$ & $58^{\mathrm{e}}$ & $60^{\mathrm{e}}$ \\
\hline Glyphosate + Chlorimuron-ethyl & $900+9$ & $52^{\mathrm{f}}$ & $76^{\mathrm{fg}}$ & $34^{\mathrm{g}}$ & $56^{\mathrm{e}}$ & $58^{\mathrm{e}}$ \\
\hline Glyphosate + Flumioxazin & $900+71$ & $61^{\mathrm{ef}}$ & $64^{\mathrm{i}}$ & $55^{\mathrm{f}}$ & $70^{\mathrm{d}}$ & $71^{\mathrm{d}}$ \\
\hline Glyphosate + Chlorimuron-ethyl + Flumioxazin & $900+9+71$ & $56^{\mathrm{ef}}$ & $70^{\mathrm{h}}$ & $68^{\mathrm{e}}$ & $73^{\mathrm{d}}$ & $73^{\mathrm{d}}$ \\
\hline
\end{tabular}

${ }^{\mathrm{z}}$ L1, LaSalle; L2, Windsor; L3, Amherstburg; L4, LaSalle; L5, Windsor; WAA, days after herbicide application; ${ }^{\mathrm{y}}$ Included merge at $1.0 \%$ vol/vol; ${ }^{\mathrm{x}}$ Included merge at $1.0 \% \mathrm{vol} / \mathrm{vol} ;{ }^{\mathrm{w}}$ Included Agral 90 at $0.25 \% \mathrm{vol} / \mathrm{vol}$ plus UAN $28 \%$; ${ }^{\mathrm{a}-\mathrm{k}}$ Means followed by the same letter are not significantly different according to fisher's protected LSD at $\mathrm{P}<0.05$.

Table 3. Percent control of glyphosate resistant giant ragweed 2 WAA for the "enhanced burndown" experiments conducted in 2011 and 2012 in Ontario, Canada.

\begin{tabular}{|c|c|c|c|c|c|c|}
\hline \multirow[b]{2}{*}{ Treatment } & \multirow[b]{2}{*}{ Rate $\left(\mathrm{g}\right.$ a.i. $\left.\mathrm{ha}^{-1}\right)$} & \multicolumn{5}{|c|}{ Percent Control $2 \mathrm{WAA}^{\mathrm{z}}$} \\
\hline & & $\mathrm{L} 1^{\mathrm{z}}$ & L2 & L3 & L4 & L5 \\
\hline Weedy Check & & $0^{\mathrm{f}}$ & $0^{\mathrm{i}}$ & $0^{\mathrm{h}}$ & $0^{\mathrm{k}}$ & $0^{\mathrm{k}}$ \\
\hline Weed Free Check & & $100^{\mathrm{a}}$ & $100^{\mathrm{a}}$ & $100^{\mathrm{a}}$ & $100^{\mathrm{a}}$ & $100^{\mathrm{a}}$ \\
\hline Glyphosate & 900 & $35^{\mathrm{e}}$ & $29^{\mathrm{h}}$ & $35^{\mathrm{g}}$ & $30^{\mathrm{j}}$ & $29^{j}$ \\
\hline Glyphosate + Amitrole & $900+2000$ & $81^{\mathrm{bc}}$ & $84^{\mathrm{de}}$ & $86^{\mathrm{cd}}$ & $78^{\mathrm{cd}}$ & $81^{\mathrm{ef}}$ \\
\hline Glyphosate $+2,4$-D Ester & $900+500$ & $94^{\mathrm{ab}}$ & $88^{\mathrm{cd}}$ & $90^{\mathrm{bc}}$ & $92^{\mathrm{b}}$ & $83^{\mathrm{de}}$ \\
\hline Glyphosate + Carfentrazone & $900+17.5$ & $74^{\mathrm{c}}$ & $49^{\mathrm{g}}$ & $73^{\mathrm{e}}$ & $74^{\text {def }}$ & $50^{\mathrm{i}}$ \\
\hline Glyphosate + Glufosinate & $900+500$ & $76^{\mathrm{c}}$ & $92^{\mathrm{c}}$ & $93^{\mathrm{b}}$ & $75^{\text {cde }}$ & $76^{\mathrm{f}}$ \\
\hline Glyphosate + Paraquat & $900+1100$ & $80^{\mathrm{bc}}$ & $98^{\mathrm{b}}$ & $86^{\mathrm{cd}}$ & $81^{\mathrm{c}}$ & $93^{\mathrm{b}}$ \\
\hline Glyphosate + Saflufenacil ${ }^{y}$ & $900+25$ & $91^{\mathrm{ab}}$ & $82^{\mathrm{e}}$ & $80^{\mathrm{de}}$ & $71^{\mathrm{efg}}$ & $90^{\mathrm{bc}}$ \\
\hline Glyphosate + Saflufenacil/Dimethenamid- $\mathrm{P}^{\mathrm{x}}$ & $900+245$ & $92^{\mathrm{ab}}$ & $89^{\mathrm{cd}}$ & $89^{\mathrm{bc}}$ & $80^{\text {cd }}$ & $87^{\text {cd }}$ \\
\hline Glyphosate + Cloransulam-methyl ${ }^{\mathrm{w}}$ & $900+17.5$ & $81^{\mathrm{bc}}$ & $85^{\mathrm{de}}$ & $76^{\mathrm{e}}$ & $68^{\mathrm{fgh}}$ & $69^{\mathrm{g}}$ \\
\hline Glyphosate + Chlorimuron-ethyl & $900+9$ & $45^{\mathrm{d}}$ & $68^{\mathrm{f}}$ & $44^{\mathrm{g}}$ & $59^{\mathrm{i}}$ & $46^{\mathrm{i}}$ \\
\hline Glyphosate + Flumioxazin & $900+71$ & $42^{\mathrm{de}}$ & $49^{\mathrm{g}}$ & $74^{\mathrm{e}}$ & $65^{\text {ghi }}$ & $63^{\mathrm{h}}$ \\
\hline Glyphosate + Chlorimuron-ethyl + Flumioxazin & $900+9+71$ & $37^{\mathrm{de}}$ & $70^{\mathrm{f}}$ & $59^{\mathrm{f}}$ & $63^{\text {hi }}$ & $69^{\mathrm{g}}$ \\
\hline
\end{tabular}

${ }^{\mathrm{z}}$ L1, LaSalle; L2, Windsor; L3, Amherstburg; L4, LaSalle; L5, Windsor; WAA, days after herbicide application; ${ }^{\mathrm{y}}$ Included merge at $1.0 \%$ vol/vol; ${ }^{\mathrm{x}}$ Included merge at $1.0 \% \mathrm{vol} / \mathrm{vol} ;{ }^{\mathrm{w}}$ Included Agral 90 at $0.25 \% \mathrm{vol} / \mathrm{vol}$ plus UAN $28 \%$; ${ }^{\mathrm{a}-\mathrm{k}}$ Means followed by the same letter are not significantly different according to fisher's protected LSD at $\mathrm{P}<0.05$. 
Table 4. Percent control of glyphosate resistant giant ragweed 4 WAA for the "enhanced burndown" experiments conducted in 2011 and 2012 in Ontario, Canada.

\begin{tabular}{|c|c|c|c|c|}
\hline \multirow[b]{2}{*}{ Treatment } & \multirow[b]{2}{*}{ Rate $\left(\mathrm{g}\right.$ a.i. $\left.\mathrm{ha}^{-1}\right)$} & \multicolumn{3}{|c|}{ Percent Control 4 WAA $^{\mathrm{z}}$} \\
\hline & & $\mathrm{L}^{\mathrm{z}}$ & L2 and L5 & $\mathrm{L} 3$ and L4 \\
\hline Weedy Check & & $0^{\mathrm{i}}$ & $0^{\mathrm{h}}$ & $0^{\mathrm{i}}$ \\
\hline Weed Free Check & & $100^{\mathrm{a}}$ & $100^{\mathrm{a}}$ & $100^{\mathrm{a}}$ \\
\hline Glyphosate & 900 & $39^{\mathrm{gh}}$ & $34^{\mathrm{g}}$ & $26^{\mathrm{h}}$ \\
\hline Glyphosate + Amitrole & $900+2000$ & $92^{\text {cd }}$ & $90^{\mathrm{ab}}$ & $93^{\mathrm{a}}$ \\
\hline Glyphosate $+2,4$-D Ester & $900+500$ & $99^{\mathrm{ab}}$ & $98^{\mathrm{a}}$ & $99^{\mathrm{a}}$ \\
\hline Glyphosate + Carfentrazone & $900+17.5$ & $76^{\mathrm{e}}$ & $27^{\mathrm{fg}}$ & $36^{\mathrm{fg}}$ \\
\hline Glyphosate + Glufosinate & $900+500$ & $77^{\mathrm{e}}$ & $58^{\mathrm{cd}}$ & $56^{\mathrm{cd}}$ \\
\hline Glyphosate + Paraquat & $900+1100$ & $79^{\mathrm{de}}$ & $84^{\mathrm{abc}}$ & $57^{\mathrm{cd}}$ \\
\hline Glyphosate + Saflufenacil ${ }^{\mathrm{y}}$ & $900+25$ & $91^{\mathrm{cd}}$ & $60^{\mathrm{cd}}$ & $46^{\mathrm{def}}$ \\
\hline Glyphosate + Saflufenacil/Dimethenamid- $\mathrm{P}^{\mathrm{x}}$ & $900+245$ & $93^{\mathrm{bc}}$ & $50^{\mathrm{cd}}$ & $47^{\mathrm{de}}$ \\
\hline Glyphosate + Cloransulam-methyl ${ }^{\mathrm{w}}$ & $900+17.5$ & $94^{\mathrm{bc}}$ & $62^{\text {bcd }}$ & $69^{\mathrm{bc}}$ \\
\hline Glyphosate + Chlorimuron-ethyl & $900+9$ & $51^{\mathrm{fg}}$ & $36^{6^{\mathrm{ef}}}$ & $39^{\mathrm{efg}}$ \\
\hline Glyphosate + Flumioxazin & $900+71$ & $44^{\mathrm{gh}}$ & $34^{\mathrm{efg}}$ & $42^{\mathrm{efg}}$ \\
\hline Glyphosate + Chlorimuron-ethyl + Flumioxazin & $900+9+71$ & $41^{\mathrm{h}}$ & $46^{\mathrm{de}}$ & $35^{\mathrm{g}}$ \\
\hline
\end{tabular}

${ }^{\mathrm{z}} \mathrm{L} 1$, LaSalle; L2, Windsor; L3, Amherstburg; L4, LaSalle; L5, Windsor; WAA, days after herbicide application; ${ }^{\mathrm{y}}$ Included Merge at 1.0\% vol/vol; ${ }^{\mathrm{x}}$ Included merge at $1.0 \% \mathrm{vol} / \mathrm{vol}$; ${ }^{\mathrm{w}}$ Included Agral 90 at $0.25 \% \mathrm{vol} / \mathrm{vol}$ plus UAN $28 \%$; ${ }^{\text {a-h }}$ Means followed by the same letter are not significantly different according to fisher's protected LSD at $\mathrm{P}<0.05$.

mid-p at $245 \mathrm{~g}$ a.i. ha $^{-1}$, respectively applied alone 4 weeks after corn emergence. Glyphosate plus carfentrazone, glufosinate, chlorimuron-ethyl, flumioxazin, or chlorimuron-ethyl plus flumioxazin provided up to $76 \%$, $77 \%, 51 \%, 44 \%$ and $46 \%$ control, respectively (Table 4).

For giant ragweed shoot dry weight all data were combined and analyzed. Glyphosate reduced giant ragweed shoot dry weight by $13 \%$ (Table 5). Glyphosate plus chlorimuron-ethyl, flumioxazin or chlorimuron-ethyl plus flumioxazin reduced giant ragweed shoot dry weight by $24 \%, 35 \%$ and $40 \%$ respectively and were equivalent to glyphosate applied alone (Table 5). In contrast, glyphosate plus 2,4-D ester or amitrole reduced giant ragweed shoot dry weight by $100 \%$ and were equivalent to the weed free control (Table 5). Glyphosate plus carfentrazone reduced giant ragweed shoot dry weight by $43 \%$ (Table 5). This is consistent with the findings of Vink et al. [15] who reported glyphosate plus carfentrazone applied at $900 \mathrm{~g}$ a.e ha ${ }^{-1}+17.5 \mathrm{~g}$ a.i ha ${ }^{-1}$ reduced giant ragweed shoot dry weight by $20 \%$ to $44 \%$. Glyphosate plus cloransulam-methyl reduced giant ragweed shoot dry weight by only $68 \%$ (Table 5). This is in contrast to Vink et al. [15] who reported $85 \%$ to $100 \%$ reduction in giant ragweed shoot dry weight with this herbicide combination. The reduced control observed in this study may be due to multiple resistant giant ragweed. Glyphosate plus paraquat, glufosinate, saflufenacil or saflufenacil/ diamethenamid-P reduced giant ragweed shoot dry weight by $82 \%, 74 \%, 66 \%$ and $71 \%$, respectively (Table 5).

For soybean yield L1, L2, and L4 were combined and L3 and L5 were combined and analyzed. Giant ragweed interference caused a reduction in soybean yield of $43 \%$ to $87 \%$ across all sites (Table 5). Giant ragweed treated with glyphosate caused a $34 \%$ to $86 \%$ reduction in soybean yield which was and was equivalent to the weedy control (Table 5). In contrast, soybean yield was equivalent to the weed free control when glyphosate plus 2,4-D ester or amitrole was applied for giant ragweed control (Table 5). In a previous study, there was no reduction in soybean yield with glyphosate plus 2,4-D ester applied at $900 \mathrm{~g}$ a.e $\mathrm{h}^{-1}+500 \mathrm{~g}$ a.i $\mathrm{h}^{-1}$ [15]. Soybean yield was not reduced due to giant ragweed interference when glyphosate plus glufosinate, paraquat, saflufenacil/dimethenamid-p or cloransulam-methyl at L1, L2 and L4 (Table 5). At L3 and L5, soybean yield with glyphosate plus glufosinate, paraquat, saflufenacil/dimethenamid-p or cloransulam-methyl was reduced by $77 \%, 75 \%, 70 \%$ and $68 \%$, respectively (Table 5). Giant ragweed interference with glyphosate plus carfentrazone, saflufenacil, chlorimuron-ethyl, flumioxazin, or chlorimuron-ethyl plus flumioxazin reduced soybean yield by up to $83 \%, 75 \%$, 
Table 5. Glyphosate resistant giant ragweed shoot dry weight and soybean yield for the "enhanced burndown" experiments conducted in 2011 and 2012 in Ontario, Canada.

\begin{tabular}{|c|c|c|c|c|}
\hline \multirow[b]{2}{*}{ Treatment } & \multirow[b]{2}{*}{ Rate } & \multirow{2}{*}{$\begin{array}{c}\text { Giant ragweed shoot dry weight } \\
\text { All combined }\end{array}$} & \multicolumn{2}{|c|}{ Soybean yield } \\
\hline & & & $\mathrm{L} 1, \mathrm{~L} 2$ and $\mathrm{L} 4^{\mathrm{z}}$ & L3 and L5 \\
\hline & $\left(\mathrm{g}\right.$ a.i. $\left.\mathrm{ha}^{-1}\right)$ & $\left(g \cdot m^{-2}\right)$ & \multicolumn{2}{|c|}{$\left(\mathrm{t} \cdot \mathrm{ha}^{-1}\right)$} \\
\hline Weedy Check & & $39.7^{\mathrm{i}}$ & $1.24^{\mathrm{ef}}$ & $0.42^{\mathrm{c}}$ \\
\hline Weed Free Check & & $0.0^{\mathrm{a}}$ & $2.19^{\mathrm{a}}$ & $3.15^{\mathrm{a}}$ \\
\hline Glyphosate & & $34.6^{\mathrm{ef}}$ & $1.35^{\mathrm{def}}$ & $0.45^{\mathrm{c}}$ \\
\hline Glyphosate + Amitrole & $900+2000$ & $1.0^{\mathrm{a}}$ & $2.08^{\mathrm{ab}}$ & $2.42^{\mathrm{a}}$ \\
\hline Glyphosate $+2,4$-D Ester & $900+500$ & $0.1^{\mathrm{a}}$ & $2.03^{\mathrm{ab}}$ & $3.37^{\mathrm{a}}$ \\
\hline Glyphosate + Carfentrazone & $900+17.5$ & $22.6^{\mathrm{cd}}$ & $1.40^{\text {cdef }}$ & $0.52^{\mathrm{bc}}$ \\
\hline Glyphosate + Glufosinate & $900+500$ & $10.5^{\mathrm{b}}$ & $1.69^{\text {abcde }}$ & $0.71^{\mathrm{bc}}$ \\
\hline Glyphosate + Paraquat & $900+1100$ & $7.1^{\mathrm{b}}$ & $1.84^{\mathrm{abcd}}$ & $0.79^{\mathrm{bc}}$ \\
\hline Glyphosate + Saflufenacil $^{\mathrm{y}}$ & $900+25$ & $13.6^{\mathrm{bc}}$ & $1.47^{\text {cdef }}$ & $0.80^{\mathrm{bc}}$ \\
\hline Glyphosate + Saflufenacil/Dimethenamid- $\mathrm{P}^{\mathrm{x}}$ & $900+245$ & $11.5^{\mathrm{b}}$ & $1.69^{\mathrm{abcde}}$ & $0.93^{\mathrm{b}}$ \\
\hline Glyphosate + Cloransulam-methyl ${ }^{\mathrm{w}}$ & $900+17.5$ & $12.8^{\mathrm{b}}$ & $1.90^{\mathrm{abc}}$ & $1.00^{\mathrm{b}}$ \\
\hline Glyphosate + Chlorimuron-ethyl & $900+9$ & $30.3^{\text {def }}$ & $1.56^{\text {bcdef }}$ & $0.62^{\mathrm{bc}}$ \\
\hline Glyphosate + Flumioxazin & $900+71$ & $25.8^{\mathrm{de}}$ & $1.03^{\mathrm{f}}$ & $0.57^{\mathrm{bc}}$ \\
\hline Glyphosate + Chlorimuron-ethyl + Flumioxazin & $900+9+71$ & $23.9^{\text {de }}$ & $1.42^{\text {cdef }}$ & $0.55^{\mathrm{bc}}$ \\
\hline
\end{tabular}

${ }^{\mathrm{z}}$ L1, LaSalle; L2, Windsor; L3, Amherstburg; L4, LaSalle; L5, Windsor; ${ }^{\mathrm{y}}$ Included merge at 1.0\% vol/vol; ${ }^{\mathrm{x}}$ Included merge at $1.0 \%$ vol/vol; ${ }^{\mathrm{w}}$ Included Agral 90 at $0.25 \% \mathrm{vol} / \mathrm{vol}$ plus UAN $28 \% ;{ }^{\mathrm{a}-\mathrm{i}}$ Means followed by the same letter are not significantly different according to fisher's protected LSD at P $<0.05$.

$80 \%, 82 \%$, and $83 \%$ and were equivalent to the weedy control (Table 5). The reduction in yields with these herbicides is consistent with control ratings and giant ragweed shoot dry weight.

\subsection{Burndown plus Residual}

The herbicides evaluated in the burndown plus residual experiment did not cause soybean injury (data not shown).

For control 1 WAA, data was statistically analyzed for each site separately except for L4 and L5 which were combined and analyzed. One WAA all the herbicides evaluated provided better control of glyphosate resistant giant ragweed compared to glyphosate alone. Control with glyphosate ranged from $35 \%$ to $65 \%$ (Table 6). Glyphosate plus saflufenacil/dimethenamid-provided $90 \%$ to $95 \%$ control and was the most effective treatment 1 WAA. For L3, control with saflufenacil/dimethenamid$\mathrm{p}$ was equivalent to the weed free control (Table 6). At 1 WAA, glyphosate plus linuron provided $61 \%$ to $90 \%$ control which is consistent with the findings of Vink et al. [15]. At 1 WAA glyphosate plus pyroxasulfone/flumioxazin, flumioxazin plus chlorimuron-ethyl, or flumi- oxazin provided up to 95,87 and 89 control, respectively (Table 6). Glyphosate plus metribuzin or cloransulammethyl provided 52 to $79 \%$ and $54 \%$ to $78 \%$ control, respectively (Table 6). This is similar to the findings of Vink et al. [15] who reported that glyphosate plusmetribuzin or cloransulam-methyl provided $55 \%$ to $70 \%$ and $56 \%$ to $88 \%$ control, respectively 1 WAA. Glyphosate plus S-metolachlor plus metribuzin or imazethapyr plus metribuzin provided $57 \%$ to $72 \%$ and $47 \%$ to $70 \%$ control, respectively (Table 6). Glyphosate plus-chlorimuron-ethyl, flumetsulam, imazethapyr, or clomazone provided up to $75 \%, 71 \%, 76 \%$ and $71 \%$ control, respectively (Table 6).

For control 2 WAA, all data were analyzed separately. Control was generally higher with all herbicides evaluated for group L1 compared to L2, L3, L4 and L5 and may be due to smaller giant ragweed (up to $9 \mathrm{~cm}$ in height) at the time of application and lower giant ragweed density (Table 7). Glyphosate provided 23\% to $42 \%$ control 2 WAA (Table 7). Glyphosate plus linuron provided $72 \%$ to $97 \%$ control across all sites (Table 7). For L2, glyphosate plus linuron provided giant ragweed control which was equivalent to the weed free control which is consistent with previous findings [15]. Gly- 
Table 6. Percent control of glyphosate resistant giant ragweed 1 WAA for the "burndown plus residual" experiments conducted in 2011 and 2012 in Ontario, Canada.

\begin{tabular}{|c|c|c|c|c|c|}
\hline \multirow[b]{2}{*}{ Treatment } & \multirow[b]{2}{*}{ Rate (g a.e./ai ha $\left.{ }^{-1}\right)$} & \multicolumn{4}{|c|}{ Percent Control 1 WAA $^{z}$} \\
\hline & & $\mathrm{L}^{\mathrm{z}}$ & $\mathrm{L} 2$ & L3 & L4 and L5 \\
\hline Weedy Check & & $0^{\mathrm{h}}$ & $0^{\mathrm{h}}$ & $0^{\mathrm{i}}$ & $0^{\mathrm{k}}$ \\
\hline Weed Free Check & & $100^{\mathrm{a}}$ & $100^{\mathrm{a}}$ & $100^{\mathrm{a}}$ & $100^{\mathrm{a}}$ \\
\hline Glyphosate & 900 & $65^{\mathrm{f}}$ & $39^{\mathrm{g}}$ & $41^{\mathrm{h}}$ & $35^{\mathrm{j}}$ \\
\hline Glyphosate + Chlorimuron-ethyl & $900+9$ & $51^{\mathrm{g}}$ & $75^{\mathrm{cde}}$ & $54^{\mathrm{ef}}$ & $46^{\mathrm{i}}$ \\
\hline Glyphosate + Chloransulam-methyl & $900+35$ & $78^{\mathrm{de}}$ & $78^{\mathrm{c}}$ & $54^{\mathrm{e}}$ & $57^{\text {gh }}$ \\
\hline Glyphosate + Linuron & $900+2250$ & $68^{\mathrm{f}}$ & $90^{\mathrm{b}}$ & $61^{\mathrm{d}}$ & $73^{\mathrm{e}}$ \\
\hline Glyphosate + Metribuzin & $900+1120$ & $79^{d}$ & $71^{\text {def }}$ & $52^{\mathrm{f}}$ & $60^{\mathrm{fg}}$ \\
\hline Glyphosate + Flumetsulam & $900+70$ & $65^{\mathrm{f}}$ & $71^{\text {def }}$ & $59^{\mathrm{de}}$ & $65^{\mathrm{f}}$ \\
\hline Glyphosate + Imazethapyr & $900+100$ & $70^{\text {ef }}$ & $76^{\mathrm{cd}}$ & $56^{\mathrm{ef}}$ & $67^{\mathrm{ef}}$ \\
\hline Glyphosate + Clomazone & $900+846$ & $66^{\mathrm{f}}$ & $71^{\mathrm{ef}}$ & $59^{\mathrm{de}}$ & $60^{\mathrm{fg}}$ \\
\hline Glyphosate + Flumioxazin & $900+71.4$ & $89^{\mathrm{c}}$ & $71^{\text {def }}$ & $74^{\mathrm{c}}$ & $80^{\mathrm{d}}$ \\
\hline Glyphosate + Flumioxazin + Chlorimuron-ethyl & $900+71.4+9$ & $87^{\mathrm{c}}$ & $76^{\text {cde }}$ & $77^{\mathrm{c}}$ & $81^{\text {cd }}$ \\
\hline Glyphosate + Pyroxasulfone + Flumioxazin & $900+240$ & $95^{\mathrm{b}}$ & $75^{\mathrm{cde}}$ & $89^{\mathrm{b}}$ & $86^{\mathrm{c}}$ \\
\hline Glyphosate + Salflufenacil/Dimethenamid-P & $900+245$ & $95^{\mathrm{b}}$ & $90^{\mathrm{b}}$ & $95^{\mathrm{ab}}$ & $91^{\mathrm{b}}$ \\
\hline Glyphosate + S-Metolachlor + Metribuzin & $900+1600+653$ & $72^{\text {def }}$ & $71^{\text {def }}$ & $57^{\mathrm{def}}$ & $64^{\mathrm{fg}}$ \\
\hline Glyphosate + Imazethapyr + Metribuzin & $900+100+400$ & $70^{\text {ef }}$ & $68^{\mathrm{f}}$ & $47^{\mathrm{g}}$ & $52^{\text {hi }}$ \\
\hline
\end{tabular}

${ }^{\mathrm{z}}$ L1, LaSalle; L2, Windsor; L3, Amherstburg; L4, LaSalle; L5, Windsor, WAA, days after herbicide application; ${ }^{\text {a- }}$ Means followed by the same letter are not significantly different according to fisher's protected LSD at $\mathrm{P}<0.05$.

Table 7. Percent control of glyphosate resistant giant ragweed 2 WAA for the "burndown plus residual" experiments conducted in 2011 and 2012 in Ontario, Canada.

\begin{tabular}{|c|c|c|c|c|c|c|}
\hline \multirow[b]{2}{*}{ Treatment } & \multirow[b]{2}{*}{ Rate (g a.e./ai ha ${ }^{-1}$ ) } & \multicolumn{5}{|c|}{ Percent Control 2 WAA $^{\mathrm{z}}$} \\
\hline & & $\mathrm{L}^{\mathrm{z}}$ & L2 & L3 & L4 & L5 \\
\hline Weedy Check & & $0^{\mathrm{i}}$ & $0^{\mathrm{g}}$ & $0^{j}$ & $0^{\mathrm{i}}$ & $0^{\mathrm{k}}$ \\
\hline Weed Free Check & & $100^{\mathrm{a}}$ & $100^{\mathrm{a}}$ & $100^{\mathrm{a}}$ & $100^{\mathrm{a}}$ & $100^{\mathrm{a}}$ \\
\hline Glyphosate & 900 & $42^{\mathrm{h}}$ & $23^{\mathrm{f}}$ & $39^{\mathrm{i}}$ & $31^{\mathrm{h}}$ & $30^{\mathrm{j}}$ \\
\hline Glyphosate + Chlorimuron-ethyl & $900+9$ & $32^{\mathrm{h}}$ & $51^{\mathrm{e}}$ & $50^{\mathrm{h}}$ & $47^{\mathrm{g}}$ & $45^{\mathrm{i}}$ \\
\hline Glyphosate + Chloransulam-methyl & $900+35$ & $88^{\text {bcd }}$ & $74^{\mathrm{b}}$ & $87^{\mathrm{b}}$ & $72^{\mathrm{bc}}$ & $68^{\mathrm{efg}}$ \\
\hline Glyphosate + Linuron & $900+2250$ & $75^{\mathrm{ef}}$ & $97^{\mathrm{a}}$ & $72^{\mathrm{cd}}$ & $79^{\mathrm{b}}$ & $94^{\mathrm{b}}$ \\
\hline Glyphosate + Metribuzin & $900+1120$ & $94^{\mathrm{b}}$ & $57^{\mathrm{de}}$ & $57^{\mathrm{fg}}$ & $57^{\mathrm{ef}}$ & $57^{\mathrm{h}}$ \\
\hline Glyphosate + Flumetsulam & $900+70$ & $62^{\mathrm{fg}}$ & $75^{\mathrm{bc}}$ & $67^{\mathrm{de}}$ & $55^{\mathrm{f}}$ & $63^{\text {fgh }}$ \\
\hline Glyphosate + Imazethapyr & $900+100$ & $80^{\mathrm{de}}$ & $74^{\mathrm{bc}}$ & $64^{\mathrm{def}}$ & $62^{\mathrm{de}}$ & $70^{\text {ef }}$ \\
\hline Glyphosate + Clomazone & $900+846$ & $66^{\mathrm{fg}}$ & $57^{\mathrm{de}}$ & $61^{\mathrm{efg}}$ & $66^{\mathrm{cd}}$ & $71^{\mathrm{ef}}$ \\
\hline Glyphosate + Flumioxazin & $900+71.4$ & $65^{\mathrm{fg}}$ & $49^{\mathrm{e}}$ & $65^{\text {def }}$ & $67^{\mathrm{cd}}$ & $76^{\mathrm{de}}$ \\
\hline Glyphosate + Flumioxazin + Chlorimuron-ethyl & $900+71.4+9$ & $60^{\mathrm{g}}$ & $57^{\mathrm{de}}$ & $72^{\mathrm{cd}}$ & $69^{c}$ & $81^{\mathrm{cd}}$ \\
\hline Glyphosate + Pyroxasulfone + Flumioxazin & $900+240$ & $89^{\mathrm{bcd}}$ & $62^{\mathrm{d}}$ & $72^{\mathrm{cd}}$ & $80^{\mathrm{b}}$ & $88^{\mathrm{bc}}$ \\
\hline Glyphosate + Salflufenacil/Dimethenamid-P & $900+245$ & $92^{\mathrm{bc}}$ & $80^{\mathrm{b}}$ & $79^{\mathrm{bc}}$ & $79^{\mathrm{b}}$ & $82^{\text {cd }}$ \\
\hline Glyphosate + S-Metolachlor + Metribuzin & $900+1600+653$ & $84^{\text {cde }}$ & $66^{\mathrm{cd}}$ & $55^{\mathrm{gh}}$ & $71^{\mathrm{c}}$ & $58^{\mathrm{gh}}$ \\
\hline Glyphosate + Imazethapyr + Metribuzin & $900+100+400$ & $75^{\mathrm{ef}}$ & $65^{\mathrm{cd}}$ & $59^{\mathrm{fg}}$ & $68^{\mathrm{cd}}$ & $65^{\mathrm{fgh}}$ \\
\hline
\end{tabular}

${ }^{{ }^{z}}$ 1, LaSalle; L2, Windsor; L3, Amherstburg; L4, LaSalle; L5, Windsor; WAA, days after herbicide application; ${ }^{a-k}$ Means followed by the same letter are not significantly different according to fisher's protected LSD at $\mathrm{P}<0.05$. 
phosate plus saflufenacil/dimethenamid-P provided 79\% to $92 \%$ control and was consistent with control ratings 1 WAA. Glyphosate plus metribuzin or cloransulam-methyl provided $57 \%$ to $94 \%$ and $68 \%$ to $88 \%$ control respectively (Table 7). At 2 WAA glyphosate plus pyroxasulfone/flumioxazin, flumioxazin plus chlorimuronethyl, S-metolachlor plus metribuzin provided up to $89 \%$, 81\%, 84\% control, respectively (Table 7). Glyphosate plus chlorimuron-ethyl, flumetsulam, imazethapyr, clomazone, flumioxazin and imazethapyr plus metribuzin provided less than $80 \%$ control (Table 7).

At 4 WAA L2 and L5 could be combined and L1, L3 and L4 were analyzed separately. Glyphosate provided $19 \%$ to $45 \%$ control 4 WAA (Table 8). Glyphosate plus linuron provided $51 \%$ to $98 \%$ control (Table 8). At L2 and L5 giant ragweed control with glyphosate plus linuron was equal to the weed free control (Table 8). Glyphosate plus cloransulam-methyl provided $73 \%$ to $94 \%$ control (Table 8). This is in contrast to the findings of Stachler [17] who reported that glyphosate plus cloransulam applied at $0.840 \mathrm{kga}$.i. ha ${ }^{-1}+0.018 \mathrm{~kg}$ a.i. $\mathrm{ha}^{-1}$ provided $69 \%$ control of glyphosate resistant giant ragweed 3 WAA. Glyphosate plus saflufenacil/dime- thenamid-p provided $51 \%$ to $89 \%$ control (Table 8). Soltani et al. [16] reported $53 \%$ to $63 \%$ control of giant ragweed with saflufenacil/dimethenamid-P at $245 \mathrm{~g}$ a.i. $\mathrm{ha}^{-1} 4$ weeks after corn emergence. Glyphosate plus metribuzin provided $25 \%$ to $91 \%$ control (Table 8). Vink et al. [15] reported $69 \%$ to $96 \%$ control with glyphosate plus metribuzin applied at $900 \mathrm{~g}$ a.i ha ${ }^{-1}+1120 \mathrm{~g}$ a.i. $\mathrm{ha}^{-1} 4$ WAA. Glyphosate plus pyroxasulfone/flumioxazin, S-metolachlor + metribuzin or imazethapyr + metribuzin provided up to $87 \%, 83 \%$ and $81 \%$ control, respectively at group L1 (Table 8). At L3, L4 and L2/L5 these herbicides provided less than $60 \%$ control. Glyphosate plus chlorimuron-ethyl, flumetsulam, imazethapyr, clomazone, flumioxazin or chlorimuron-ethyl plus flumioxazin provided less than $80 \%$ control (Table 8).

At 8 WAA L4 and L5 could be combined and L1, L2 and L3 were analyzed separately. Glyphosate provided less than 31\% control across all sites 8 WAA (Table 9). Glyphosate plus linuron provided $23 \%$ to $99 \%$ control (Table 9). At L2, giant ragweed control with glyphosate plus linuron was equal to the weed free control but it was not commercially acceptable at other locations (Table 9). This is in contrast to Vink et al. [15] who reported 95\%

Table 8. Percent control of glyphosate resistant giant ragweed 4 WAA for the "burndown plus residual" experiments conducted in 2011 and 2012 in Ontario, Canada.

\begin{tabular}{|c|c|c|c|c|c|}
\hline \multirow[b]{2}{*}{ Treatment } & \multirow[b]{2}{*}{ Rate (g a.e./ai ha ${ }^{-1}$ ) } & \multicolumn{4}{|c|}{ Percent Control 4 WAA $^{\mathrm{z}}$} \\
\hline & & $\mathrm{L}^{\mathrm{z}}$ & $\mathrm{L} 2$ and L5 & L3 & L4 \\
\hline Weedy Check & & $0^{\mathrm{k}}$ & $0^{\mathrm{k}}$ & $0^{\mathrm{i}}$ & $0^{\mathrm{k}}$ \\
\hline Weed Free Check & & $100^{\mathrm{a}}$ & $100^{\mathrm{a}}$ & $100^{\mathrm{a}}$ & $100^{\mathrm{a}}$ \\
\hline Glyphosate & 900 & $45^{\mathrm{j}}$ & $22^{\mathrm{j}}$ & $31^{\mathrm{h}}$ & $19^{j}$ \\
\hline Glyphosate + Chlorimuron-ethyl & $900+9$ & $36^{\mathrm{j}}$ & $35^{\mathrm{ij}}$ & $40^{\mathrm{efg}}$ & $28^{\mathrm{fgh}}$ \\
\hline Glyphosate + Chloransulam-methyl & $900+35$ & $94^{\mathrm{b}}$ & $76^{\mathrm{bc}}$ & $92^{\mathrm{a}}$ & $73^{\mathrm{b}}$ \\
\hline Glyphosate + Linuron & $900+2250$ & $76^{\mathrm{fgh}}$ & $98^{\mathrm{a}}$ & $51^{\text {bcd }}$ & $69^{\mathrm{bc}}$ \\
\hline Glyphosate + Metribuzin & $900+1120$ & $91^{\mathrm{bc}}$ & $52^{\text {efgh }}$ & $33^{\mathrm{fgh}}$ & $25^{\mathrm{h}}$ \\
\hline Glyphosate + Flumetsulam & $900+70$ & $66^{\mathrm{hi}}$ & $54^{\mathrm{efg}}$ & $41^{\operatorname{defg}}$ & $26^{\mathrm{g}}$ \\
\hline Glyphosate + Imazethapyr & $900+100$ & $78^{\text {efgh }}$ & $54^{\mathrm{efg}}$ & $52^{\mathrm{bc}}$ & $32^{\mathrm{fgh}}$ \\
\hline Glyphosate + Clomazone & $900+846$ & $69^{\text {ghi }}$ & $71^{\mathrm{cd}}$ & $60^{\mathrm{b}}$ & $53^{\mathrm{cd}}$ \\
\hline Glyphosate + Flumioxazin & $900+71.4$ & $70^{\text {ghi }}$ & $36^{\mathrm{hij}}$ & $41^{\text {def }}$ & $34^{\mathrm{efg}}$ \\
\hline Glyphosate + Flumioxazin + Chlorimuron-ethyl & $900+71.4+9$ & $61^{\mathrm{i}}$ & $53^{\mathrm{efg}}$ & $49^{\text {bcde }}$ & $30^{\mathrm{fgh}}$ \\
\hline Glyphosate + Pyroxasulfone + Flumioxazin & $900+240$ & $87^{\text {bcde }}$ & $58^{\text {def }}$ & $47^{\text {cde }}$ & $42^{\mathrm{de}}$ \\
\hline Glyphosate + Salflufenacil/Dimethenamid-P & $900+245$ & $89^{\mathrm{bcd}}$ & $64^{\text {cde }}$ & $51^{\mathrm{bcd}}$ & $51^{\mathrm{d}}$ \\
\hline Glyphosate + S-Metolachlor + Metribuzin & $900+1600+653$ & $83^{\text {cdef }}$ & $37^{\text {ghi }}$ & $32^{\mathrm{gh}}$ & $33^{\text {efgh }}$ \\
\hline Glyphosate + Imazethapyr + Metribuzin & $900+100+400$ & $81^{\text {defg }}$ & $47^{\text {fghi }}$ & $47^{\text {cde }}$ & $35^{\mathrm{ef}}$ \\
\hline
\end{tabular}

${ }^{\mathrm{z}}$ L1, LaSalle; L2, Windsor; L3, Amherstburg; L4, LaSalle; L5, Windsor; WAA, days after herbicide application; ${ }^{\mathrm{a}-\mathrm{k}}$ Means followed by the same letter are not significantly different according to fisher's protected LSD at $\mathrm{P}<0.05$. 
Table 9. Percent control of glyphosate resistant giant ragweed 8 WAA for the "burndown plus residual" experiments conducted in 2011 and 2012 in Ontario, Canada.

\begin{tabular}{|c|c|c|c|c|c|}
\hline \multirow[b]{2}{*}{ Treatment } & \multirow[b]{2}{*}{ Rate (g a.e./ai ha ${ }^{-1}$ ) } & \multicolumn{4}{|c|}{ Percent Control 8 WAA $^{z}$} \\
\hline & & $\mathrm{L}^{\mathrm{z}}$ & L2 & L3 & L4 and L5 \\
\hline Weedy Check & & $0^{\mathrm{g}}$ & $0^{\mathrm{f}}$ & $0^{\mathrm{e}}$ & $0^{\mathrm{f}}$ \\
\hline Weed Free Check & & $100^{\mathrm{a}}$ & $100^{\mathrm{a}}$ & $100^{\mathrm{a}}$ & $100^{\mathrm{a}}$ \\
\hline Glyphosate & 900 & $31^{\mathrm{f}}$ & $12^{\mathrm{d}}$ & $9^{\text {cd }}$ & $10^{\mathrm{e}}$ \\
\hline Glyphosate + Chlorimuron-ethyl & $900+9$ & $35^{\mathrm{f}}$ & $12^{\mathrm{d}}$ & $9^{\text {cd }}$ & $9^{\mathrm{e}}$ \\
\hline Glyphosate + Chloransulam-methyl & $900+35$ & $88^{\mathrm{abc}}$ & $45^{\mathrm{b}}$ & $90^{\mathrm{a}}$ & $51^{\mathrm{bc}}$ \\
\hline Glyphosate + Linuron & $900+2250$ & $73^{\mathrm{de}}$ & $99^{\mathrm{a}}$ & $23^{\mathrm{b}}$ & $68^{\mathrm{b}}$ \\
\hline Glyphosate + Metribuzin & $900+1120$ & $93^{\mathrm{ab}}$ & $13^{\mathrm{d}}$ & $8^{\mathrm{cd}}$ & $21^{\text {de }}$ \\
\hline Glyphosate + Flumetsulam & $900+70$ & $66^{\mathrm{e}}$ & $35^{\mathrm{bc}}$ & $12^{\mathrm{bc}}$ & $12^{\mathrm{e}}$ \\
\hline Glyphosate + Imazethapyr & $900+100$ & $70^{\mathrm{de}}$ & $17^{\mathrm{cd}}$ & $10^{\mathrm{cd}}$ & $13^{\mathrm{e}}$ \\
\hline Glyphosate + Clomazone & $900+846$ & $63^{\mathrm{e}}$ & $16^{\mathrm{d}}$ & $10^{\mathrm{cd}}$ & $32^{\mathrm{cd}}$ \\
\hline Glyphosate + Flumioxazin & $900+71.4$ & $65^{\mathrm{e}}$ & $11^{\mathrm{d}}$ & $10^{\mathrm{cd}}$ & $13^{\mathrm{e}}$ \\
\hline Glyphosate + Flumioxazin + Chlorimuron-ethyl & $900+71.4+9$ & $65^{\mathrm{e}}$ & $13^{\mathrm{d}}$ & $13^{\mathrm{bc}}$ & $19^{\mathrm{de}}$ \\
\hline Glyphosate + Pyroxasulfone + Flumioxazin & $900+240$ & $76^{\text {cde }}$ & $11^{\mathrm{d}}$ & $8^{\mathrm{cd}}$ & $23^{\mathrm{de}}$ \\
\hline Glyphosate + Salflufenacil/Dimethenamid-P & $900+245$ & $83^{\mathrm{bcd}}$ & $11^{\mathrm{d}}$ & $7^{\mathrm{cd}}$ & $32^{\text {cd }}$ \\
\hline Glyphosate + S-Metolachlor + Metribuzin & $900+1600+653$ & $77^{\text {cde }}$ & $5^{\mathrm{e}}$ & $5^{\mathrm{d}}$ & $14^{\mathrm{e}}$ \\
\hline Glyphosate + Imazethapyr + Metribuzin & $900+100+400$ & $72^{\mathrm{de}}$ & $12^{\mathrm{d}}$ & $7^{\mathrm{cd}}$ & $16^{\mathrm{de}}$ \\
\hline
\end{tabular}

${ }^{\mathrm{z}}$ L1, LaSalle; L2, Windsor; L3, Amherstburg; L4, LaSalle; L5, Windsor; WAA, days after herbicide application; ${ }^{\mathrm{a}-\mathrm{g}}$ Means followed by the same letter are not significantly different according to fisher's protected LSD at $\mathrm{P}<0.05$.

to $98 \%$ control 8 WAAwith glyphosate plus linuron applied at $900 \mathrm{~g}$ a.e. ha ${ }^{-1}+2250 \mathrm{~g}$ a.i. $\mathrm{ha}^{-1}$. Glyphosate plus cloransulam-methyl provided $45 \%$ to $90 \%$ control (Table 9). At L1 and L2, giant ragweed control with glyphosate plus cloransulam-methyl was equivalent to the weed free control. Vink et al. [15] reported glyphosate plus cloransulam-methyl at $900 \mathrm{~g}$ a.e. $\mathrm{ha}^{-1}+35 \mathrm{~g}$ a.i. ha $^{-1}$ provided $75 \%$ to $95 \%$ control 8 WAA. Glyphosate plus metribuzin provided $8 \%$ to $93 \%$ control (Table 9). At L1, giant ragweed control with glyphosate plus metribuzin was equal to the weed free control. Vink et al. [15] reported glyphosate plus metribuzin provided $60 \%$ to $71 \%$ control 8 WAA applied at $900 \mathrm{~g}$ a.e. $\mathrm{ha}^{-1}+$ $1120 \mathrm{~g}$ a.i. $\mathrm{ha}^{-1}$. Glyphosate plus saflufenacil/dimethenamid-P provided $7 \%$ to $83 \%$ control (Table 9). Soltani et al. [16] reported $43 \%$ to $57 \%$ control with saflufenacil/ dimethenamid-p applied alone at $735 \mathrm{~g}$ a.i. ha ${ }^{-1} 8$ WAA. Glyphosate plus chlorimuron-ethyl, flumetsulam, imazethapyr, or clomazone provided up to $35 \%, 66 \%, 70 \%$ and $63 \%$ control, respectively which are consistent with earlier ratings (Table 9). Glyphosate plus flumioxazin, chlorimuron-ethyl plus flumioxazin, pyroxasulfone/flumioxazin, S-metolachlor plus metribuzin or imazethapyr plus metribuzin provided less than $80 \%$ control across all locations (Table 9).

For giant ragweed shoot dry weight L2, L3, L4, and L5 could be combined while L1 was analyzed separately.There was a greater reduction in giant ragweed shoot dry weight at L1 (Table 10). All herbicides with the exception of glyphosate reduced giant ragweed shoot dry weight by $55 \%$ or greater at L1 (Table 10). Glyphosate reduced giant ragweed shoot dry weight by $46 \%$ to $66 \%$ across all locations (Table 10). At L1, giant ragweed shoot dry weight with glyphosate was equivalent to the weedy control. In contrast, glyphosate plus linuron reduced giant ragweed shoot dry weight by $93 \%$ to $94 \%$ across all locations (Table 10). This is similar to the findings of Vink et al. [15] who reported a 99\% reduction in giant ragweed shoot dry weight with glyphosate plus linuron applied at $900 \mathrm{~g}$ a.e. $\mathrm{ha}^{-1}+2250 \mathrm{~g}^{\mathrm{a}}$.i. ha ${ }^{-1}$. Glyphosate plus cloransulam-methyl or metribuzin reduced giant ragweed shoot dry weight by $82 \%$ to $100 \%$ and $49 \%$ to $100 \%$, respectively (Table 10). At L1, giant ragweed shoot dry weight with glyphosate plus cloransulam-methyl or metribuzin was equivalent to the weed free control. This is similar to the findings of Vink et al. 
Table 10. Glyphosate resistant giant ragweed shoot dry weight and soybean yield for the "burndown plus residual" experiments conducted in 2011 and 2012 in Ontario, Canada.

\begin{tabular}{|c|c|c|c|c|c|}
\hline \multirow[b]{2}{*}{ Treatment } & \multirow[b]{2}{*}{ Rate } & \multicolumn{2}{|c|}{ Giant ragweed shoot dry weight } & \multicolumn{2}{|c|}{ Soybean yield } \\
\hline & & $\mathrm{L}^{\mathrm{z}}$ & L2, L3, L4 and L5 & L1 & L2, L3, L4 and L5 \\
\hline & $\left(\mathrm{g}\right.$ a.e./ai ha $\left.{ }^{-1}\right)$ & & $\left.g \cdot m^{-2}\right)$ & & $\left.h a^{-1}\right)$ \\
\hline Weedy Check & & $27.1^{\mathrm{j}}$ & $59.0^{\mathrm{h}}$ & $0.35^{\mathrm{g}}$ & $0.40^{\text {cd }}$ \\
\hline Weed Free Check & & $0.0^{\mathrm{a}}$ & $0.0^{\mathrm{a}}$ & $1.76^{\mathrm{a}}$ & $2.79^{\mathrm{a}}$ \\
\hline Glyphosate & 900 & $9.3^{\text {ghij }}$ & $31.7^{\mathrm{eg}}$ & $0.55^{\mathrm{efg}}$ & $0.47^{\mathrm{cd}}$ \\
\hline Glyphosate + Chlorimuron-ethyl & $900+9$ & $12.2^{\mathrm{ij}}$ & $43.0^{\mathrm{g}}$ & $0.48^{\mathrm{fg}}$ & $0.61^{\mathrm{c}}$ \\
\hline Glyphosate + Chloransulam-methyl & $900+35$ & $0.0^{\mathrm{ab}}$ & $10.8^{\mathrm{bc}}$ & $1.58^{\mathrm{ab}}$ & $1.07^{\mathrm{b}}$ \\
\hline Glyphosate + Linuron & $900+2250$ & $2.0^{\text {bcdef }}$ & $3.6^{\mathrm{b}}$ & $1.05^{\mathrm{c}}$ & $1.32^{\mathrm{b}}$ \\
\hline Glyphosate + Metribuzin & $900+1120$ & $0.0^{\mathrm{abc}}$ & $30.0^{\operatorname{defg}}$ & $1.60^{\mathrm{a}}$ & $0.45^{\mathrm{cd}}$ \\
\hline Glyphosate + Flumetsulam & $900+70$ & $9.4^{\mathrm{hij}}$ & $27.7^{\operatorname{defg}}$ & $0.98^{\mathrm{cd}}$ & $0.63^{\mathrm{c}}$ \\
\hline Glyphosate + Imazethapyr & $900+100$ & $3.4^{\text {defgh }}$ & $38.0^{\mathrm{fg}}$ & $0.62^{\text {defg }}$ & $0.40^{\text {cd }}$ \\
\hline Glyphosate + Clomazone & $900+846$ & $6.4^{\mathrm{fghi}}$ & $23.0^{\text {def }}$ & $0.61^{\text {defg }}$ & $0.53^{\text {cd }}$ \\
\hline Glyphosate + Flumioxazin & $900+71.4$ & $5.3^{\text {efghi }}$ & $37.1^{\mathrm{eg}}$ & $0.68^{\text {cdef }}$ & $0.43^{\text {cd }}$ \\
\hline Glyphosate + Flumioxazin + Chlorimuron-ethyl & $900+71.4+9$ & $5.1^{\text {efghi }}$ & $25.4^{\text {def }}$ & $0.61^{\text {defg }}$ & $0.55^{\mathrm{c}}$ \\
\hline Glyphosate + Pyroxasulfone + Flumioxazin & $900+240$ & $2.7^{\text {cdefg }}$ & $22.3^{\text {de }}$ & $0.59^{\text {defg }}$ & $0.50^{\text {cd }}$ \\
\hline Glyphosate + Salflufenacil/Dimethenamid-P & $900+245$ & $1.1^{\mathrm{abcd}}$ & $17.1^{\mathrm{cd}}$ & $1.07^{\mathrm{bc}}$ & $0.67^{\mathrm{c}}$ \\
\hline Glyphosate + S-Metolachlor + Metribuzin & $900+1600+653$ & $1.4^{\text {bcde }}$ & $37.0^{\mathrm{eg}}$ & $0.89^{\text {cde }}$ & $0.29^{\mathrm{d}}$ \\
\hline Glyphosate + Imazethapyr + Metribuzin & $900+100+400$ & $2.7^{\text {cdef }}$ & $30.3^{\text {defg }}$ & $0.58^{\mathrm{efg}}$ & $0.44^{\text {cd }}$ \\
\hline
\end{tabular}

${ }^{\mathrm{z}}$ L1, LaSalle; L2, Windsor; L3, Amherstburg; L4, LaSalle; L5, Windsor; ${ }^{\mathrm{a}-\mathrm{j}}$ Means followed by the same letter are not significantly different according to fisher's protected LSD at $\mathrm{P}<0.05$.

[15] who reported a $97 \%$ and $95 \%$ reduction in giant ragweed shoot dry weight with glyphosate plus cloransulam-methyl or metribuzin, respectively. Giant ragweed shoot dry weight was reduced by $71 \%$ to $96 \%$ with glyphosate plus saflufenacil/dimethenamid-P and was equivalent to the weed free control at L1 (Table 10). In contrast, glyphosate plus chlorimuron-ethyl or flumetsulam reduced giant ragweed shoot dry weight by $27 \%$ to $55 \%$ and $53 \%$ to $65 \%$ and was equivalent to the weedy control at group L1 (Table 10). Glyphosate plus S-metolachlor plus metribuzin, imazethapyr plus metribuzin, pyroxasulfone/flumioxazin, flumioxazin plus chlorimuron-ethyl, flumioxazin, clomazone or imazethapyr, reduced giant ragweed biomass by less than $65 \%$ at L2, L3, L4 and L5 (Table 10).

Soybean yield at L2, L3, L4 and L5 could be combined and L1 was analyzed separately. Giant ragweed interference caused a reduction in soybean yield by $80 \%$ to $86 \%$ across all sites (Table 10). Baysinger and Sims [14] reported a $92 \%$ yield loss in soybean with a giant ragweed density of 16 plants per $9 \mathrm{~m}$ of row. Giant ragweed interference with all herbicides had a reduction in soybean yield greater than 5\% (Table 10). Giant ragweed interference with glyphosate alone caused a $69 \%$ to $83 \%$ reduction in soybean yield (Table 10). Giant ragweed interference with glyphosate plus cloransulam-methyl or metribuzin caused a $10 \%$ to $62 \%$ and $9 \%$ to $84 \%$ reduction in soybean yield, respectively and was equivalent to the weed free control at L1 (Table 10). Giant ragweed interference with glyphosate plus linuron caused a $40 \%$ to $53 \%$ reduction in soybean yield which is in contrast to the reduction in giant ragweed shoot dry weight (Table 10). Giant ragweed interference with glyphosate plus chlorimuron-ethyl, flumetsulam, imazethapyr, clomazone, flumioxazin, chlorimuron-ethyl plus flumioxazin, pyroxasulfone plus flumioxazin, saflufenacil/dimethenamid-P, S-metolachlor plus metribuzin or imazethapyr plus metribuzion caused greater than $35 \%$ reduction in soybean yield across all locations (Table 10).

\section{CONCLUSION}

In summary, glyphosate plus 2,4-D ester or amitrole provided the best control of glyphosate resistant giant ragweed. Herbicides providing residual activity provided 
variable control across all locations. Out of the burndown plus residual herbicides evaluated, glyphosate plus linuron provided the best control of glyphosate resistant giant ragweed. Although this herbicide provided the highest control of glyphosate resistant giant ragweed, control was not always acceptable across all locations. This research shows that control of glyphosate resistant giant ragweed is needed early in the season when plants are small. Future research should look at other herbicide tankmixes with multiple modes of action.

\section{ACKNOWLEDGEMENTS}

The authors acknowledge Chris Kramer for his expertise and technical assistance in these studies. Funding for this project was provided in part by Monsanto Canada Inc., the Grain Farmers of Ontario and the Agricultural Adaptation Council through the Canadian Agricultural Adaptation Program.

\section{REFERENCES}

[1] Amrhein, N., Schab, J. and Steinrücken, H.C. (1980) The mode of action of the herbicide glyphosate. Naturwissenschaften, 67, 356-357. doi:10.1007/BF01106593

[2] Franz, J.E., Mao, M.K. and Sikorski, J.A. (1997) Glyphosate: A unique global herbicide. American Chemical Society, Washington DC.

[3] Sprankle, P., Meggitt, W.F. and Penner, D. (1975) Rapid inactivation of glyphosate in the soil. Weed Science, 23, 224-228.

[4] Dill, G.M., Sammons, R.D., Feng, P.C.C., Kohn, F., Kretzmer, K., Mehrsheikh, A., Bleeke, M., Honegger, J.L., Farmer, D., Wright, D. and Haupfear, E.A. (2010) Glyphosate: Discovery, development, applications, and properties. In: Nandula, V.K., Ed., Glyphosate Resistance in Crops and Weeds: History, Development, and Management, John Wiley and Sons, Inc., Hoboken, 1-33. doi:10.1002/9780470634394.ch1

[5] Dill, G.M., CaJacob, C.A. and Padgette, S.R. (2008) Glyphosate resistant crops: Adoption, use and future considerations. Pest Management Science, 64, 326-331. doi:10.1002/ps.1501

[6] Owen, M.D.K. (2008) Weed species shifts in glyphosate resistant crops. Pest Management Science, 64, 377-387. doi:10.1002/ps. 1539

[7] Bradshaw, L., Padgette, D.S.R., Kimball, S.L. and Wells, B.H. (1997) Perspectives on glyphosate resistance. Weed Technology, 11, 189-198.

[8] Powles, S.B., Lorraine-Colwill, D.F., Dellow, J.J. and Preston, C. (1998) Evolved resistance to glyphosate in rigid ryegrass (Loliumrigidum) in Australia. Weed Science, 46, 604-607.

[9] Heap, I. (2012) International survey of herbicide resistant weeds. http://www.weedscience.org/In.asp

[10] Bassett, I.J. and Crompton, C.W. (1982) The biology of Canadian weeds.: 55.: Ambrosia trifida L. Canadian Journal of Plant Science, 62, 1003-1010. doi:10.4141/cjps82-148

[11] Harrison, S.K., Regnier, E.E., Schmoll, J.T. and Webb, J.E. (2001) Competition and fecundity of giant ragweed in corn. Weed Science, 49, 224-229. doi:10.1614/0043-1745(2001)049[0224:CAFOGR]2.0.C $\underline{\mathrm{O} ; 2}$

[12] Schutte, B.J., Regnier, E.E. and Harrison, S.K. (2008) The association between seed size and seed longevity among maternal families in Ambrosia trifida L. populations. Seed Science Research, 18, 201-211. doi:10.1017/S0960258508082974

[13] Johnson, B., Loux, M., Nordby, D., Sprague, C., Nice, G., Westhoven, A. and Stachler, J. (2007) Biology and management of giant ragweed. http://www.ces.purdue.edu/extmedia/BP/GWC-12.pdf

[14] Baysinger, J.A. and Sims, B.D. (1992) Giant ragweed (Ambrosia trifida) control in soybean (Glycine max). Weed Technology, 6, 13-18.

[15] Vink, J.P., Soltani, N., Robinson, D.E., Tardif, F.J., Lawton, M.B. and Sikkema, P.H. (2012) Glyphosate-resistant giant ragweed (Ambrosia trifida L.) control with preplant herbicides in soybean (Glycine max L.). Canadian Journal of Plant Science, 92, 913-922. doi:10.4141/cjps2012-025

[16] Soltani, N., Shropshire, C. and Sikkema, P.H. (2011) Giant ragweed (Ambrosia trifida L.) control in corn. Canadian Journal of Plant Science, 91, 577-581. doi:10.4141/cjps2010-004

[17] Stachler, J.M. (2008) Characterization and management of glyphosate-resistant giant ragweed (Ambrosia trifida L.) and horseweed [Conyzacanadensis (L.) Cronq.]. Ph.D. Dissertation, The Ohio State University, Columbus, 60-107. 